\title{
AURANTIPORUS CROCEUS, A FLAGSHIP SPECIES OF THE EUROPEAN FUNGAL CONSERVATION IS RE-DISCOVERED AFTER HALF CENTURY IN HUNGARY
}

\author{
Attila Koszka ${ }^{\text {\& Viktor Papp }}{ }^{*}$ \\ ${ }^{1}$ Hungarian Mycological Society, Budapest, Hungary; ${ }^{2}$ Department of Botany, Szent \\ István University, 1518 Budapest, Hungary; \\ *E-mail: agaricum@gmail.com
}

\begin{abstract}
The threatened polypore, Aurantiporus croceus has previously been known only from one locality in Hungary, and the basidiome of this species has not been seen in the country since 1972. In this study, a new Hungarian finding of $A$. croceus is reported from an old-growth forest reserve in the Vértes Mts (Central Transdanubia). We present the nrDNA ITS sequence, macro- and microscopical characteristics as well as photographs of the new specimen.
\end{abstract}

Keywords: Central Europe, Hapalopilus, Meruliaceae, phylogeny, Polyporales, Quercus

\section{INTRODUCTION}

The peculiar polypore species, Aurantiporus croceus (Pers.) Murrill (三 Hapalopilus croceus (Pers.) Bondartsev \& Singer) is easily recognised in the field by the often large sized, bright orange-red coloured basidiocarps growing predominantly on veteran oak (Quercus) trees. Aurantiporus croceus is widely distributed in Europe (Ryvarden and Melo 2014) and also reported from the eastern parts of Asia (Dai 2012) and eastern North America (Zhou et al. 2016). Despite its widespread distribution and easily recognisable basidiome, $A$. croceus is considered to be a rare species everywhere. As a result of its rarity and its spectacular appearance, it was placed in the spotlight of the European fungal conservation; it is listed amongst the 33 threatened fungi proposed to be included in the Bern Convention (Dahlberg and Croneborg 2003), and red-listed in 12 European countries (listed as CR in eight countries), and included in nine regional Red Data Books of Russia (Dahlberg 2019). Due to the significantly declined 
population, it became very rare and scattered, it has recently been assessed to the IUCN's Red List (Dahlberg 2019).

From Hungary, only historical specimens of A. croceus were known. These are originated from an old living Quercus tree located near Sitke, Western Transdanubia (Igmándy 1968, Szabó 2012). Despite the single locality of this rare polypore, it was not included in the proposed Hungarian Red List (Rimóczi et al. 1999). Considering the specific habitat preference and the rarity of $A$. croceus in Hungary, the second author urged to protect this species by law, which was achieved in 2013 (MK 2013). However, despite the greater attention, no new occurrence of this species was observed until 2018. During a mycological survey of Juhdöglö-völgy Forest Reserve (Vértes Mts, Central Transdanubia) in late May, a new location of A. croceus was found. In this study the ITS sequence, macro- and microscopical characteristics and photographs of the new Hungarian specimen of $A$. croceus are presented.

\section{MATERIALS AND METHODS}

\section{Isolates and morphology}

The new Hungarian specimen was deposited in the private herbarium of the authors. We report the macromorphological descriptions based on field notes. Micromorphological data were obtained from the dried specimens, which were observed under a Zeiss Axio Imager.A2 light microscope, equipped with AxioVision Release 4.8.2. software. Measurements were done with a $100 \times$ oil immersion objective (1000x magnification). Observations of microscopic features as well as measurements were made on slide preparations stained with Melzer's reagent. Spores were measured by cutting sections from the tubes. The following abbreviations were used in the description of the basidiospores: IKI = Melzer's reagent, IKI- = both inamyloid and indextrinoid, $\mathrm{L}=$ mean spore length, $\mathrm{W}=$ mean spore width, $\mathrm{Q}=$ variation in the $\mathrm{L} / \mathrm{W}$ ratios, $\mathrm{n}=$ number of measured spores.

\section{Molecular study}

Primers ITS1F and ITS4 (White et al. 1990, Gardes and Bruns 1993) were used to amplify the ITS (internal transcribed spacer) region of the nuclear ribosomal DNA. For the amplification we used the 
Phire ${ }^{\circledR}$ Plant Direct PCR Kit (Thermo Scientific, USA) following the manufacturer's recommendations. The PCR (polymerase chain reaction) protocols were set according to Papp and Dima (2017). The quality of PCR products were checked in 2\% agarose gels. The amplicons were sequenced commercially at the Biological Research Centre (Szeged, Hungary) with the same primers used in the PCR reactions. The chromatograms were checked, assembled and edited with the CodonCodeAligner 7.0.1 (CodonCode Corporation, Centerville, MA, USA).

The newly generated Aurantiporus croceus sequence is deposited in GenBank (Benson et al. 2017); the accession numbers are included in Table 1. For the phylogenetic analysis, similar sequences were searched from GenBank using the BLASTn search tool (Altschul et al. 1990). The ITS region was aligned with PRANK (Löytynoja and Goldman 2005, 2008) as implemented in its graphical interface (PRANKSTER) using default settings. SeaView 4 (Gouy et al. 2010) was used to visually inspect and improve the alignment. The nucleotide dataset resulted an alignment length of 697 characters. The dataset was subjected to maximum likelihood (ML) and Bayesian inference (BI) phylogenetic analyses, which were performed in raxmlGUI (Silvestro and Michalak 2012) and MrBayes 3.1.2 (Ronquist and Huelsenbeck 2003), respectively. ML analysis was done using 1000 rapid bootstrap searches. For the nucleotide partition the GTRGAMMA substitution model, while for the indel partition the RAxML default set for binary characters were applied. In the BI analysis the GTR + G model of evolution for the nucleotide partition, and the two-parameter Markov model (Mk2 Lewis) for the indel partition were applied. The BI settings were: four Markov chain Monte Carlo (MCMC) over 5 million generations, sampling every 1000th generation, two independent runs, and burn-in of 20\% (the first 1000 trees were discarded). Post burn-in trees were used to compute a 50\% majority rule consensus phylogram. Phylogenetic trees from both ML and BI analyses resulted in congruent topologies. The best scoring tree from the ML analysis was edited with MEGA6 (Tamura et al. 2013) and presented in Figure 1. 
Table 1. Details of the specimens comprised in this study. Species, herbarium voucher numbers, country, and GenBank accession numbers are presented.

\begin{tabular}{|c|c|c|c|c|}
\hline Species & $\begin{array}{l}\text { Specimen } \\
\text { voucher }\end{array}$ & $\begin{array}{l}\text { Collection } \\
\text { site }\end{array}$ & $\begin{array}{l}\text { GenBank } \\
\text { number }\end{array}$ & References \\
\hline $\begin{array}{l}\text { Aurantipileus } \\
\text { mayanensis }\end{array}$ & TJB10228, type & Belize & HM772140 & GiNNS et al. (2010) \\
\hline A. mayanensis & JV 1504/128 & Costa Rica & KT156706 & unpublished \\
\hline $\begin{array}{l}\text { Aurantiporus } \\
\text { croceus }^{1}\end{array}$ & BRNM 737561 & $\begin{array}{l}\text { Czech } \\
\text { Republic }\end{array}$ & JQ821320 & DvořÁK et al. (2014) \\
\hline A. croceus $^{1}$ & $\mathrm{H} 6-27$ & Lithuania & MH571407 & unpublished \\
\hline A. croceus & VPapp 300518-1 & Hungary & MT876120 & this study \\
\hline $\begin{array}{l}\text { Geesterania } \\
\text { carneola }\end{array}$ & MCW 474/13 & Brazil & KY175000 & $\begin{array}{l}\text { WESTPHALEN } \text { et al. } \\
(2018)\end{array}$ \\
\hline G. carneola & MCW 388/12 & Brazil & KY174999 & $\begin{array}{l}\text { WESTPHALEN et al. } \\
(2018)\end{array}$ \\
\hline G. davidii & $\begin{array}{l}\text { MCW 396/12, } \\
\text { type }\end{array}$ & Brazil & KY174998 & $\begin{array}{l}\text { WESTPHALEN } \text { et al. } \\
\text { (2018) }\end{array}$ \\
\hline G. davidii & MCW 370/12 & Brazil & KY174997 & $\begin{array}{l}\text { WESTPHALEN } \text { et al. } \\
\text { (2018) }\end{array}$ \\
\hline $\begin{array}{l}\text { Luteoporia } \\
\text { albomarginata }\end{array}$ & Dai 15229, type & China & KU598873 & Wu et al. (2016) \\
\hline L. albomarginata & GC $1702-1$ & Taiwan & MF496147 & CHEN et al. (2018a) \\
\hline L. albomarginata & TNM GC 1702-1 & Taiwan & LC379003 & CHEN et al. (2018b) \\
\hline $\begin{array}{l}\text { Odoria } \\
\text { alborubescens }\end{array}$ & $\begin{array}{l}\text { PC } 0706595, \\
\text { type }\end{array}$ & France & MG097863 & PAPP \& DIMA (2018) \\
\hline O. alborubescens & BР 106943 & Hungary & MG097864 & PAPP \& DIMA (2018) \\
\hline O. alborubescens ${ }^{2}$ & BRNU 627479 & $\begin{array}{l}\text { Czech } \\
\text { Republic }\end{array}$ & JQ821319 & DvoŘÁK et al. (2014) \\
\hline Pappia fissilis $^{3}$ & $\begin{array}{l}\text { SFC20140626- } \\
03\end{array}$ & $\begin{array}{l}\text { South } \\
\text { Korea }\end{array}$ & KX792915 & KIM et al. (2016) \\
\hline P. fissilis ${ }^{3}$ & BRNM 699803 & $\begin{array}{l}\text { Czech } \\
\text { Republic }\end{array}$ & HQ728292 & ToMŠOVSKÝ (2012) \\
\hline P. fissilis ${ }^{3}$ & HHB9530sp & USA & KY948774 & Justo et al. (2017) \\
\hline $\begin{array}{l}\text { Phlebiporia } \\
\text { bubalina }\end{array}$ & Dai 13168, type & China & KC782526 & Chen \& Cui (2014) \\
\hline P. bubalina & Dai 15231 & China & KU598876 & Wu et al. (2016) \\
\hline P. bubalina & Dai 9798 & China & KY131842 & Wu et al. (2017) \\
\hline $\begin{array}{l}\text { Hapalopilus } \\
\text { rutilans }\end{array}$ & JV0407/34J & USA & KX752619 & $\begin{array}{l}\text { MiETTINEN et al. } \\
(2016)\end{array}$ \\
\hline H. rutilans & Н 6012735 & Finland & KX752614 & $\begin{array}{l}\text { MIETTINEN et al. } \\
\text { (2016) }\end{array}$ \\
\hline
\end{tabular}

${ }^{1}$ as Hapalopilus; ${ }^{2}$ as Aurantiporus; ${ }^{3}$ as Tyromyces 


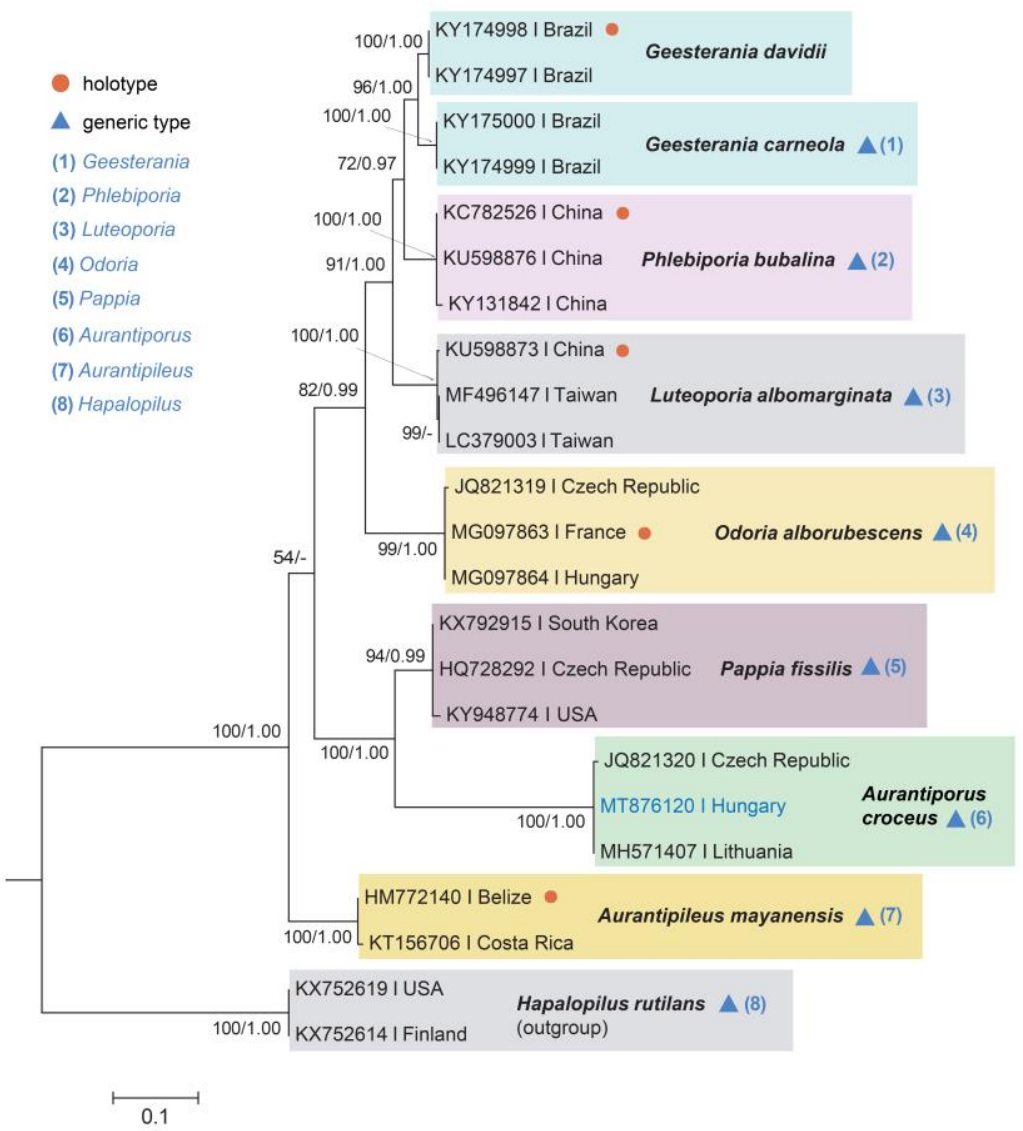

Figure 1. Phylogenetic tree of Aurantiporus croceus and related poroid species in Meruliaceae inferred from MrBayes and RAxML analyses of the nrDNA ITS sequences based on the best scoring maximum likelihood (ML) tree. Hapalopilus rutilans served for outgroup. Bayesian posterior probabilities (PP) $>0.9$ and ML bootstrap values $>50 \%$ as evidences of statistical support are shown above or below branches. The bar indicates 0.1 expected change per site per branch.

\section{RESULTS}

\section{ITS sequence analyses}

The dataset represents 21 sequences of eight poroid Meruliaceae species, with Hapalopilus rutilans as outgroup. The Hungarian specimen (GenBank: MT876120) cluster together with other $A$. croceus specimens (labelled as Hapalopilus croceus) collected in 
Czech Republic (GenBank: JQ821320) and Lithuania (GenBank: MH571407). The sequences of other morphologically similar European species formerly discussed in Aurantiporus (viz. Odoria alborubescens (Bourdot \& Galzin) V. Papp \& Dima and Pappia fissilis (Berk. \& M.A. Curtis) Zmitr.) cluster in well-separated strongly supported clades, respectively.

\section{Taxonomy}

Aurantiporus croceus (Pers.) Murrill, Mycologia 12(1): 11, 1920

(Figures 2-3)

$\equiv$ Boletus croceus Pers., Observationes mycologicae 1: 87, 1796; $\equiv$ Polyporus croceus (Pers.) Fr., Observ. mycol. (Havniae) 1: 124, 1815; 三 Phaeolus croceus (Pers.) Pat., Essai taxonomique sur les familles et les genres des Hyménomycètes: 86, 1900; 三 Hapalopilus croceus (Pers.) Bondartsev \& Singer, Annales Mycologici 39 (1): 52, 1941; 三 Tyromyces croceus (Pers.) J. Lowe, Mycotaxon 2 (1): 21, 1975

= Polyporus pilotae Schwein., Transactions of the American Philosophical Society 4 (2): 156,1832

Basidiocarps annual, occasionally semi-perennial (Figure 3), broadly attached, pileate; upper surface bright orange-yellow at first, and finely pubescent, becomes vivid orange later, finely brownish orange and almost smooth when old; flesh vivid orange, dark wine-red to almost black when touched with KOH. Context soft and watery when fresh, shrinking considerably and becomes hard and rigid when dry, taste sourish or slightly bitter. Pore surface bright reddish-orange when fresh, brownish when dry, pores angular, 2-3 per mm. Tubes 2-3 cm thick, bright orange, spongiose and watery when fresh, drying darker orange to brownish, becomes hard and resinous. Hyphal system monomitic, hyphae hyaline and thin-walled, moderately branched, 3-6 $\mu \mathrm{m}$ in diam., septa with clamps. Hyphae richly encrusted with golden yellow crystals, forming a dense and loose covering around the wall. Yellow incrustation layer lose its elements in small pieces easily. Basidia 4-spored, 18-30 × 7-10 $\mu \mathrm{m}$, clamped. Cystidia or other sterile elements absent. Basidiospores broadly ellipsoid, (4.02-) 4.12-4.36(-4.48) x (2.82-)3.00-3.22(-3.26) $\mu \mathrm{m}, \mathrm{L}=4.25$ $\mu \mathrm{m}, \mathrm{W}=3.1 \mu \mathrm{m}, \mathrm{Q}=1.37(\mathrm{n}=30)$, hyaline, thin-walled, smooth, negative in Melzer's reagent.

Specimens examined: HUNGARY. Vas County, near Sitke, Bajti, on old living Quercus robur, leg. Z. Igmándy, Pagony et Varga, 14 Sept 1964 (Igmándy 1488); leg. Haracsi et Igmándy, 17 Sept 1965 
(Igmándy 1599), leg. Z. Igmándy et Varga, 3 Dec 1966 (Igmándy 1675); leg. Z. Igmándy, 8 Nov 1968 (Igmándy 1807); leg. Z. Igmándy, 28 Oct 1969 (Igmándy 1848); leg. Z. Igmándy, 11 Oct 1972 (Igmándy 2010); Fejér County, Vértes Mts, near Csákberény, Juhdöglő-völgy Forest Reserve, on the underside of large Quercus sp. log, leg. A. Koszka et V. Papp, 30 May 2018 (VPapp 300518-1), GenBank: MT876120. CZECH REPUBLIC. Moravia, on Quercus robur, leg. A. Černý, 2 May 1958 (Igmándy 10129).
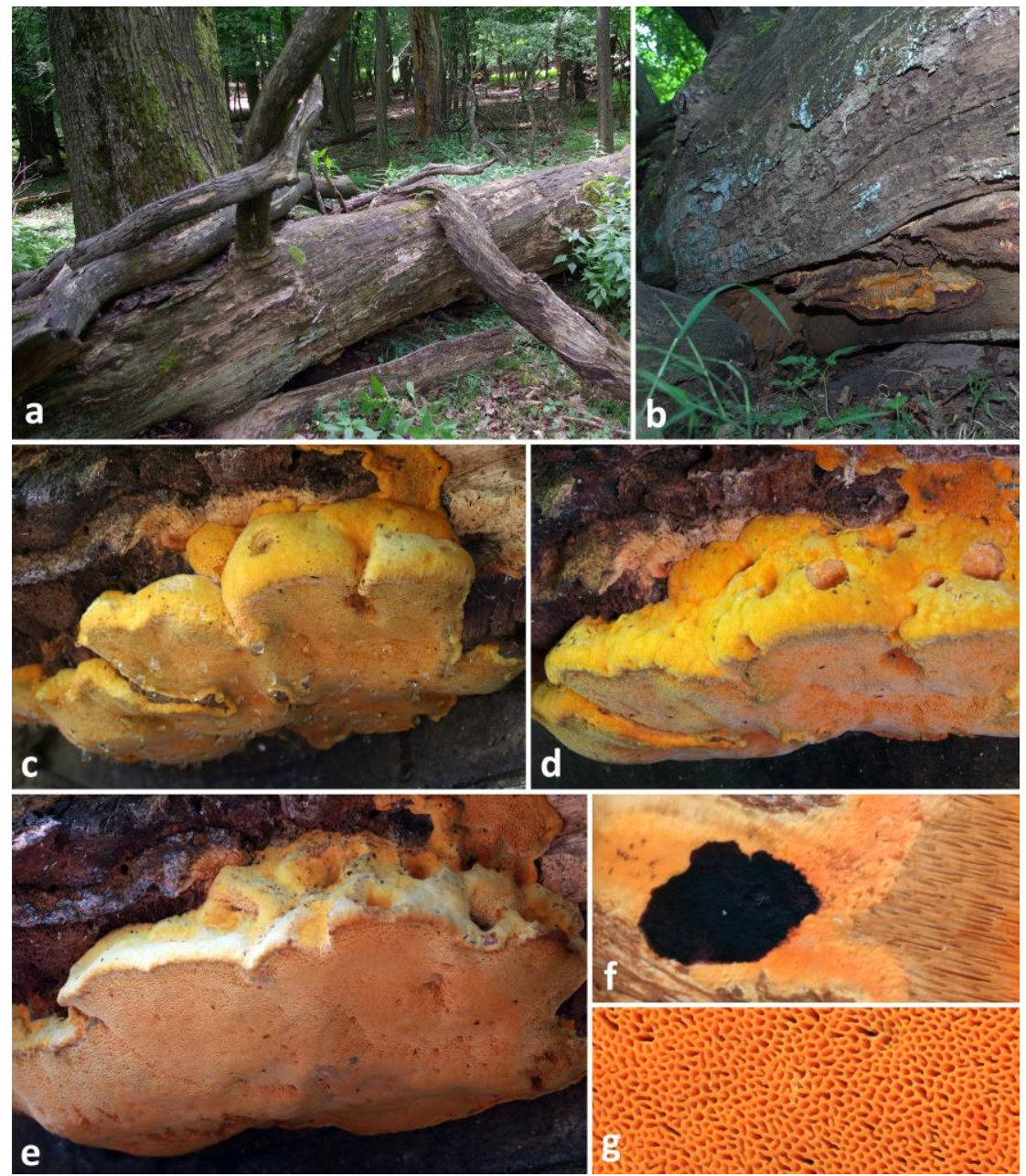

Figure 2. Macromorphology and habitat of Aurantiporus croceus. a-b: habitat in Juhdöglővölgy Forest Reserve. c-e: basidiomata. f: KOH reaction of the basidiomata. g: pore surface (fresh material). Photos (a, b: V. Papp, c, e, f, g: A. Koszka, d: P. Finy). 


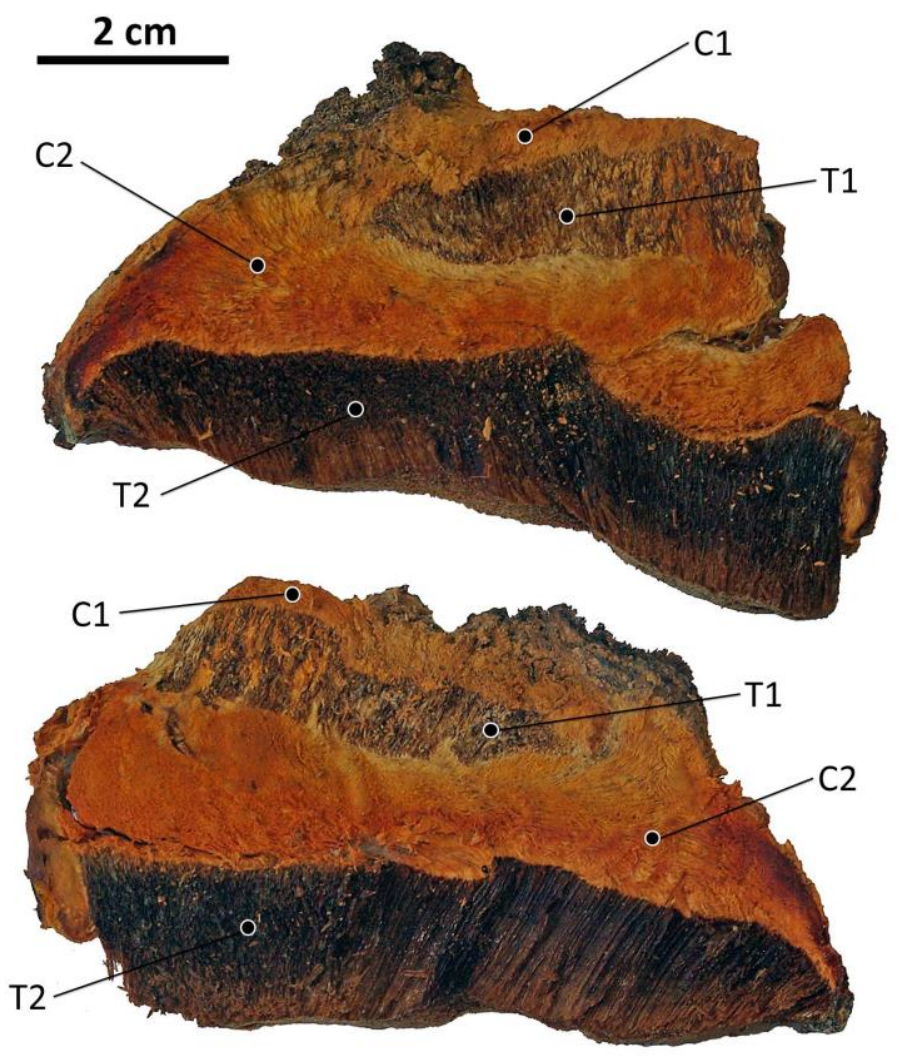

Figure 3. Cross-section of Aurantiporus croceus basidiome (dried material). C1: old context, C2: new context, T1: old trama, T2: new trama.

\section{DISCUSSION}

Most observations of $A$. croceus are originated from living, old and coarse veteran trees, mainly oak (Quercus) and more rarely on chestnut (Castanea) in parks and old growth forests (Dahlberg 2019). The new Hungarian location of $A$. croceus is reported from an old oak forest site at the Juhdöglö-völgy Forest Reserve, which is considered as one of the few European primary forests extant in 
the Pannonian Biogeographic region (Sabatini et al. 2018). The lignicolous funga of the Juhdöglö-völgy Forest Reserve have intensely been studied in the last decade, and several rare and threatened wood-inhabiting basidiomycetes were documented (Papp 2011, 2012, Papp and Szabó 2013, Papp and Dima 2014, 2018, Papp et al. 2012, 2016; Crous et al. 2018, Liu et al. 2018). Despite of the consecutive, targeted search of $A$. croceus at the old oak forest sections of the core area, only one location was observed. The basidiocarp grew 10 meters from the root zone of an old oak log (Figure $2 a, b$ ). Although Juhdöglő-völgy Forest Reserve are protected and no forest management practices are being applied on the collection site, the crowded population of boar, red deer and mouflon creates a discontinuity in the forest. Thus, the genotype (or population) of A. croceus investigated in the Juhdöglo"völgy Forest Reserve is threatened by the decreasing habitat quality.

Acknowledgement: The authors wish to thank Jenő Jakab (Institute of Sylviculture and Forest Protection, University of Sopron) for providing additional information on the specimens collected by Zoltán Igmándy.

\section{REFERENCES}

Altschul, S.F., Gish, W., Miller, W., Myers, E.W. \& Lipman, D.J. (1990). Basic local alignment search tool. Journal of Molecular Biology 215: 403-410.

https://doi.org/10.1016/S0022-2836(05)80360-2

Benson, D.A., Cavanaugh, M., Clark, K., Karsch-Mizrachi, I., Lipman, D.J., Ostell, J. \& SAYERS, E.W. (2017). GenBank. Nucleic Acids Research 45(D1): D37-D42. https://doi.org/10.1093/nar/gkw1070

Chen, J.J. \& CuI, B.K. (2014). Phlebiporia bubalina gen. et. sp. nov. (Meruliaceae, Polyporales) from Southwest China with a preliminary phylogeny based on rDNA sequences. Mycological Progress 13: 563-573. https://doi.org/10.1007/s11557-013-0940-4

Chen, C.C., Wu, S.H. \& Chen, C.Y. (2018a). Twelve species of polypores (basidiomycetes) new to Taiwan. Fungal Science 33(1): 7-19.

ChEn, C.C., WU, S.H. \& CHEN, C.Y. (2018b). Hydnophanerochaete and Odontoefibula, two new genera of phanerochaetoid fungi (Polyporales, Basidiomycota) from East Asia. MycoKeys 39: 75-96. https://doi.org/10.3897/mycokeys.39.28010

Crous, P.W., Wingfield, M.J., Burgess, T.I., Hardy, G.E.St.J., Gené, J., Guarro, J., BaSeia, I.G., García, D., Gusmão, L.F.P., Souza-Motta, C.M., Thangavel, R., AdamČík, S., Barili, A., Barnes, C.W., Bezerra, J.D.P., Bordallo, J.J., CANo-Lira, J.F., DE Oliveira, R.J.V., Ercole, E., Hubka, V., Iturrieta-González, I., Kubátová, A., Martín, M.P., Moreau, P.-A., Morte, A., Ordoñez, M.E., Rodríguez, A., Stchigel, A.M., Vizzini, A., Abdollahzadeh, J., Abreu, V.P., AdamČí́KovÁ, K., Albuquerque, G.M.R., AleXandrova, 
A.V., Álvarez Duarte, E., Armstrong-Cho, C., Banniza, S., Barbosa, R.N., Bellanger, J.-M., Bezerra, J.L., Cabral, T.S., Caboñ, M., Caicedo, E., Cantillo, T., Carnegie, A.J., Carmo, L.T., Castañeda-Ruiz, R.F., Clement, C.R., Čmoková, A., Conceição, L.B., CruZ, R.H.S.F., Damm, U., da Silva, B.D.B., Da Silva, G.A., DA Silva, R.M.F., Santiago, A.L.C.M. De A., De Oliveira, L.F., De Souza, C.A.F., DÉniel, F., DimA, B., Dong, G., Edwards, J., FÉlix, C.R., Fournier, J., Gibertoni, T.B., Hosaka, K., Iturriaga, T., Jadan, M., JanY, J.-L., JuRJević, Ž., KolaŘíK, M., KuŠAn, I., LANDEll, M.F., LeITE Cordeiro, T.R., Lima, D.X., Loizides, M., Luo, S., Machado, A.R., Madrid, H., Magalhães, O.M.C., Marinho, P., MatočEc, N., Mešíc, A., Miller, A.N., Morozova, O.V., NeVes, R.P., Nonaka, K., NovákovÁ, A., Oberlies, N.H., Oliveira-Filho, J.R.C., Oliveira, T.G.L., Papp, V., Pereira, O.L., Perrone, G., Peterson, S.W., Pham, T.H.G., Raja, H.A., Raudabaugh, D.B., IŘehulKa, J., Rodrāguez-Andrade, E., Saba, M., Schauflerovāj, A., Shivas, R.G., Simonini, G., Siqueira, J.P.Z., Sousa, J.O., StajSic, V., Svetasheva, T., Tan, Y.P., TkalČEc, Z., Ullah, S., Valente, P., Valenzuela-LoPeZ, N., Abrinbana, M., Viana Marques, D.A., Wong, P.T.W., Xavier De Lima, V. \& Groenewald, J.Z. (2018). Fungal Planet description sheets 716-784. Persoonia 40: 240-387. https://doi.org/10.3767/persoonia.2018.40.10

DAHLBERG, A. (2019). Hapalopilus croceus. The IUCN Red List of Threatened Species 2019: e.T58521209A58521216.

Dahlberg, A. \& Croneborg, H. (eds.) (2003). 33 threatened fungi in Europe. Complementary and revised information on candidates for listing in Appendix 1 of the Bern Convention. Swedish Environmental Protection Agency (EPA) and European Council for the Conservation of Fungi (ECCF), $82 \mathrm{pp}$.

DAI, Y.C. (2012). Polypore diversity in China with an annotated checklist of Chinese polypores. Mycoscience 53: 49-80. https://doi.org/10.1007/s10267-011-0134-3

DvořÁK, D., BĚŤÁK, J. \& ToмšovsкÝ, M. (2014). Aurantiporus alborubescens (Basidiomycota, Polyporales) - first record in the Carpathians and notes on its systematic position. Czech Mycology 66(1): 71-84.

https://doi.org/10.33585/cmy.66105

Gardes, M. \& BRUNS, T.D. (1993). ITS primers with enhanced specificity for basidiomycetes - application to the identification of mycorrhizae and rusts. Molecular Ecology 2(2): 113-118. https://doi.org/10.1111/j.1365-294x.1993.tb00005.x

Ginns, J., Lindner, D.L., BARONI, T.J. \& RyvaRdEN, L. (2010). Aurantiopileus mayanensis a new genus and species of polypore (Polyporales, Basidiomycota) from Belize with connections to existing Asian species. North American Fungi 5(4): 1-10. https://doi.org/10.2509/naf2010.005.004

Gouy, M., Guindon, S. \& Gascuel, O. (2010). SeaView version 4: a multiplatform graphical user interface for sequence alignment and phylogenetic tree building. Molecular Biology and Evolution 27(2): 221-224.

https://doi.org/10.1093/molbev/msp259

IGMÁnDY, Z. (1968). Die Porlinge Ungarns und ihre phytopathologische Bedeutung III. (Polipori Hungariae III.). Acta Phytopathologica Academiae Scientiarum Hungaricae 3(3): 349-359.

Justo, A., Miettinen, O., Floudas, D., Ortiz-Santana, B., Sjökvist, E., Lindner, D., Nakasone, K., Niemelä, T., Larsson, K.H., Ryvarden, L. \& Hibbett, D.S. (2017). A 
revised family-level classification of the Polyporales (Basidiomycota). Fungal Biology 121: 798-824. https://doi.org/10.1016/j.funbio.2017.05.010

Kim, N.K., PARK, J.Y., PARK, M.S., LeE, H., Cho, H.J., Eimes, J.A., Kim, C. \& Lim, Y.W. (2016). Five new wood decay fungi (Polyporales and Hymenochaetales) in Korea. Mycobiology 44(3): 146-154. https://doi.org/10.5941/myco.2016.44.3.146

Liu, L.-N., Razaq, A., Atri, N.S., Bau, T., Belbahri, L., Chenari Bouket, A., Chen, L.-P., Deng, C., Ilyas, S., Khalid, A.N., Kitaura, M.J., Kobayashi, T., Li Y., Lorenz, A.P., MA, Y.-H., Malysheva, E., Malysheva, V., Nuytinck, J., Qiao, M., Saini, M.K., Scur, M.C., Sharma, S., Shu, L.-L., Spirin, V., TANaKa, Y., Tojo, M., Uzuhashi, S., VAlério-Júnior, C., Verbeken, A., Verma, B., Wu, R.-H., Xu, J.-P., Yu, Z.-F., Zeng, H., Zhang, B., Banerjee, A., Beddiar, A., Bordallo, J.J., DAfri, A., Dima, B., Krisai-Greilhuber, I., Lorenzini, M., Mandal, R., Morte, A., NAth, P.S., Papp, V., Pavlík, J., Rodríguez, A., ŠEvč̌́íKovÁ, H., Urban, A., Voglmayr, H. \& Zapparoli, G. (2018). Fungal Systematics and Evolution: FUSE 4. Sydowia 70: 211-286.

https://doi.org/10.12905/0380.sydowia70-2018-0211

LÖYTYNOJA, A. \& Goldman, N. (2005). An algorithm for progressive multiple alignment of sequences with insertions. Proceedings of the National Academy of Sciences of the United States of America 102(30): 10557-10562.

https://doi.org/10.1073/pnas.0409137102

LÖYTYNOJA, A. \& GoldMAN, N. (2008). Phylogeny-aware gap placement prevents errors in sequence alignment and evolutionary analysis. Science 320: 16321635. https://doi.org/10.1126/science.1158395

Miettinen, O., Spirin, V., Vlasák, J., Rivoire, B., Stenroos, S. \& Hibbett, D.S. (2016). Polypores and genus concepts in Phanerochaetaceae (Polyporales, Basidiomycota). MycoKeys 17: 1-46. https://doi.org/10.3897/mycokeys.17.10153

MK (2013). 83/2013. (IX. 25.) VM rendelet. A védett és a fokozottan védett növény- és állatfajokról, a fokozottan védett barlangok köréről, valamint az Európai Közösségben természetvédelmi szempontból jelentős növény- és állatfajok közzétételéről szóló 13/2001. (V. 9.) KöM rendelet módosításáról. Magyar Közlöny 2013(156): 67479-67503. (in Hungarian)

PAPP, V. (2011). Adatok a Xylobolus nemzetség magyarországi előfordulásáról. Mikológiai Közlemények, Clusiana 50(2): 173-182. (in Hungarian)

PAPP, V. (2012). A Frantisekia mentschulensis első magyarországi előfordulása. Mikológiai Közlemények, Clusiana 51(2): 181-186. (in Hungarian)

PAPP, V. \& DiMA, B. (2014). A Pholiota squarrosoides első magyarországi előfordulása és előzetes filogenetikai vizsgálata. Mikológiai Közlemények, Clusiana 53(1-2): 33-42. (in Hungarian)

PAPP, V. \& DimA, B. (2017). Favolus gracilisporus (Polyporaceae, Basidiomycota), an East Asian polypore species new to the European mycobiota. Mycosphere 8(6): 1177-1184. https://doi.org/10.5943/mycosphere/8/6/7

PAPP, V. \& DimA, B. (2018). New systematic position of Aurantiporus alborubescens (Meruliaceae, Basidiomycota), a threatened old-growth forest polypore. Mycological Progress 17(3): 319-332.

https://doi.org/10.1007/s11557-017-1356-3

Papp, V., Dima, B., KoszkA, A. \& Siller, I. (2012). A Donkia pulcherrima (Polyporales, Basidiomycota) első magyarországi előfordulása és taxonómiai értékelése. Mikológiai Közlemények, Clusiana 53(1-2): 43-53. (in Hungarian) 
Papp, V., Király, G., Koscsó, J., Malatinszky, Á., Nagy, T., TAKÁcs, A. \& Dima, B. (2016). Taxonomical and chorological notes 2 (20-27). Studia botanica hungarica 47(1): 179-191. https://doi.org/10.17110/studbot.2016.47.1.179

PAPP, V. \& SzABó, I. (2013). Distribution and host preference of poroid basidiomycetes in Hungary I. - Ganoderma. Acta Silvatica et Lignaria Hungarica 9: 71-83. https://doi.org/10.2478/aslh-2013-0006

Rimóczi, I., Siller, I., VASAS, G., Albert, L., VetTer, J. \& BrateK, Z. (1999). Magyarország nagygombáinak javasolt vörös listája. Mikológiai Közlemények, Clusiana 38(13): 107-132. (in Hungarian)

RonQuist, F. \& HuELSENBECK, J.P. (2003). MrBayes 3: Bayesian phylogenetic inference under mixed models. Bioinformatics 19: 1572-1574.

https://doi.org/10.1093/bioinformatics/btg180

Ryvarden, L. \& Melo, I. (2014). Poroid fungi of Europe. Synopsis Fungorum 31: 1450.

Sabatini, F.M., Burrascano, S., Keeton, W.S., Levers, C., Lindner, M., Pötzschner, F., Verkerk, P.J., Bauhus, J., Buchwald, E., Chaskovsky, O., Debaive, N., Horváth, F., Garbarino, M., Grigoriadis, N., Lombardi, F., Duarte, I.M., Meyer, P., Midteng, R., Mikac, S., Mikolas, M., Motta, R., Mozgeris, G., Nunes, L., Panayotov, M., Ódor, P., Ruete, A., Simovski, B., Stillhard, J., Svoboda, M., Szwagrzyk, J., Tikkanen, O.-P., Volosyanchuk, R., Vrska, T., Zlatanov, T.M. \& Kuemmerle, T. (2018). Where are Europe's last primary forests? Diversity and Distributions 24(12): 1890-1892. https://doi.org/10.1111/ddi.12778

Silvestro, D. \& MichalaK, I. (2012). raxmlGUI: a graphical front-end for RAxML. Organisms Diversity \& Evolution 12: 335-337. https://doi.org/10.1007/s13127-011-0056-0

SzABó, I. (2012). Poroid fungi of Hungary in the collection of Zoltán Igmándy. Acta Silvatica et Lignaria Hungarica 8: 113-122. https://doi.org/10.2478/v10303-012-0009-0

Tamura, K., Stecher, G., Peterson, D., Filipski, A. \& Kumar, S. (2013). MEGA6: molecular evolutionary genetics analysis version 6.0. Molecular Biology and Evolution 30(12): 2725-2729. https://doi.org/10.1093/molbev/mst197

TомšovsкÝ, M. (2012). Delimitation of an almost forgotten species Spongipellis litschaueri (Polyporales, Basidiomycota) and its taxonomic position within the genus. Mycological Progress 11(2): 415-424. https://doi.org/10.1007/s11557-011-0756-z

Westphalen, M.C, RajChenberg, M., TomšovskÝ, M. \& Gugliotta, A.M. (2018). A reevaluation of Neotropical Junghuhnia s.lat. (Polyporales, Basidiomycota) based on morphological and multigene analyses. Persoonia 41: 130-141. https://doi.org/10.3767/persoonia.2018.41.07

White, T.J., BRUnS, T., LEE, S. \& TAYLOR, J. (1990). Amplification and direct sequencing of fungal ribosomal RNA genes for phylogenetics. In: InNIS, M.A., GELFAND, D.H., Sninsky, J.J. \& White, T.J. (eds.): PCR protocols: a guide to methods and applications. Academic Press, New York, pp. 315-332.

Wu, F., YuAn, Y., CHEN, J.J. \& HE, S.H. (2016). Luteoporia albomarginata gen. et sp. nov. (Meruliaceae, Basidiomycota) from tropical China. Phytotaxa 263(1): 31-41. https://doi.org/10.11646/phytotaxa.263.1.3 
WU, F., ChEn, J.J., JI, X.H., VlasáK, J. \& DAI, Y.C. (2017). Phylogeny and diversity of the morphologically similar polypore genera Rigidoporus, Physisporinus, Oxyporus, and Leucophellinus. Mycologia 109: 749-765.

https://doi.org/10.1080/00275514.2017.1405215

Zhou, L.W., Nakasone, K.K., Burdsall, H.H. JR, Ginns, J., VlasáK, J., Miettinen, O., Spirin, V., Niemelä, T., YuAN, H.S., He, S.H., CuI, B.K., XING, J.H. \& DAI, Y.C. (2016). Polypore diversity in North America with an annotated checklist. Mycological Progress 15: 771-790. https://doi.org/10.1007/s11557-016-1207-7

(submitted: 20.05.2020, accepted: 12.08.2020) 